Parallel Processing Letters Vol. 5 No. 4 (1995) 697-700

(C) World Scientific Publishing Company

\title{
AUTHOR INDEX \\ VOLUME 5 (1995)
}

Ahuja, M. and Raynal, M., An implementation of global flush primitives using counters

5 (1995) 171-178

Akl, S.G., see Pavel, S.

Andonov, R., Quinton, P., Rajopadhye, S. and Wilde, D., A shift register-based systolic array for the unbounded knapsack problem

Baker, J., see Merry, M.S.

Banerjee, P., see Ramaswamy, S.

Ben-Asher, Y., The cartesian product problem and implementing production systems on reconfigurable meshes

Berkman, O. and Matias, Y., Fast parallel algorithms for minimum and related problems with small integer inputs

Bhattacharya, S., Kirani, S. and Tsai, W.T., Quadtree layouts and $\mathrm{I} / \mathrm{O}$ bandwidth

Bollig, B., Hühne, M., Pölt, S. and Savický, P., On the average case circuit delay of disjunction

Bongiovanni, G., Crescenzi, P. and De Agostino, S., MAX SAT and MIN set cover approximation algorithms are $P$-complete

Brewer, E.A., see Chong, F.T.

Cai, W. and Skillicorn, D.B., Calculating recurrences using the bird-meertens formalism

Cappello, F., see Germain, C.

Chan, I.W. and Choi, F., An optimal systolic dictionary

Chandra, A. and Melhem, R., Reconfiguration in fault-tolerant $3 \mathrm{D}$ meshes

Choi, F., see Chan, I.W.

Chong, F.T., Sharma, S.D., Brewer, E.A. and Saltz, J., Multiprocessor runtime support for fine-grained, irregular DAGs

Chu, Y.-K. and Rover, D.T., An effective two-dimensional mesh partitioning strategy

Cole, M.I., Parallel programming with list homomorphisms

Collard, J.-F., Risset, T. and Feautrier, P., Construction of DO loops from systems of affine constraints

Corrêa, R. and Ferreira, A., On the effectiveness of synchronous parallel branch-and-bound algorithms

Cosnard, M. and Loi, M., Automatic task graph generation techniques

5 (1995) 205-212

5 (1995) 251-262

5 (1995) 401-412

5 (1995) $587-598$

5 (1995) 49-61

5 (1995) 223-230

5 (1995) 231-240

5 (1995) 275-280

5 (1995) 293-298

5 (1995) 671-683

5 (1995) 179-190

5 (1995) 97-109

5 (1995) 451-460

5 (1995) 387-399

5 (1995) 451-460

5 (1995) 671-683

5 (1995) 623-634

5 (1995) 191-203

5 (1995) 421-436

5 (1995) 375-386

5 (1995) 527-538 
Cosnard, M., Editorial note

Cosnard, M., Editorial note

Crescenzi, P., see Bongiovanni, G.

Darte, A. and Vivien, F., Revisiting the decomposition of Karp, Miller and Winograd

De Agostino, S., see Bongiovanni, G.

Deo, N., see Perumalla, K.

Desprez, F., Dongarra, J.J. and Tourancheau, B., Performance study of $L U$ factorization with low communication overhead on multiprocessors

Dongarra, J.J., see Desprez, F.

Drach, N., Gefflaut, A., Joubert, P. and Seznec, A., About cache associativity in low-cost shared memory multi-microprocessors

Eswar, K., see Kumar, B.

Feautrier, P., see Collard, J.-F.

Ferreira, A., see Corrêa, R.

Gao, G.R., see Ning, Q.

Gefflaut, A., see Drach, N

Gerasoulis, A. and Yang, T., Editorial note

Germain, C. and Cappello, F., The static network: A high performance reconfigurable communication network

Gupta, V. and Schenfeld, E., Task graph partitioning and mapping in a reconfigurable parallel architecture

Hagerup, T. and Keller, J., Fast parallel permutation algorithms

Haglin, D.J., Bipartite expander matching is in NC

Ho, C.-T. and Kao, M.-Y., Efficient broadcast on hypercubes with wormhole and $\mathrm{E}$-Cube routings

Huang, C.-H., see Kumar, B.

Hühne, M., see Bollig, B.

Hurson, A.R., see Lee, B.

Ibarra, L. and Richards, D., Tree open ear decomposition in parallel graph algorithms

Jayasimha, D.N., see Lutz, D.R.

Joubert, P., see Drach, N.

Kao, M.-Y., see Ho, C.-T.

Kaufmann, M., Schröder, H. and Sibeyn, J.F., Routing and sorting on reconfigurable meshes

Keller, J., see Hagerup, T.

Kirani, S., see Bhattacharya, S.

Krizanc, D. and Narayanan, L., Zero-one sorting on the mesh Kumar, B., Eswar, K., Sadayappan, P. and Huang, C.-H., A clustering algorithm for parallel sparse cholesky factorization

Lee, B. and Hurson, A.R., A strategy for scheduling partially ordered program graphs onto multicomputers

Lin, T.-H., see Wang, J.-C.

Liou, J.-C., see Palis, M.A.

Loi, M., see Cosnard, M.

Lorts, D.M., see Shirazi, B.

Lutz, D.R. and Jayasimha, D.N., Do fixed-processor communication-time tradeoffs exist?

5 (1995) 125-127

5 (1995) 329-330

5 (1995) 293-298

5 (1995) 551-562

5 (1995) 293-298

5 (1995) 367-373

5 (1995) 157-169

5 (1995) $157-169$

5 (1995) 475-487

5 (1995) 685-696

5 (1995) 421-436

5 (1995) 375-386

5 (1995) 539-550

5 (1995) 475-487

5 (1995) 525-526

5 (1995) 97-109

5 (1995) 563-574

5 (1995) 139-148

5 (1995) 413-420

5 (1995) 213-222

5 (1995) 685-696

5 (1995) 275-280

5 (1995) 575-586

5 (1995) 129-138

5 (1995) 311-320

5 (1995) 475-487

5 (1995) 213-222

5 (1995) 81-95

5 (1995) 139-148

5 (1995) 231-240

5 (1995) 149-156

5 (1995) 685-696

5 (1995) 575-586

5 (1995) 647-658

5 (1995) 635-646

5 (1995) 527-538

5 (1995) 599-610

5 (1995) 311-320 
MacKenzie, P.D., A separation between reconfigurable mesh models

Mahesh, S., Murthy, C.S.R. and Rangan, C.P., Performance bounds for static multiprocessor scheduling of multi-task jobs

Maniatty, W., see Szymanski, B.K.

Matias, Y., see Berkman, O.

Melhem, R., see Chandra, A.

Merry, M.S. and Baker, J., A constant time sorting algorithm for a three dimensional reconfigurable mesh and reconfigurable network

Miller, R. and Schuster, A., Editorial note

Murthy, C.S.R., see Mahesh, S.

Nakano, K., A bibliography of published papers on dynamically reconfigurable architectures

Nakano, K., Prefix-sums algorithms on reconfigurable meshes

Narayanan, L., see Krizanc, D.

Niedermeier, R. and Rossmanith, P., On optimal OROW-PRAM algorithms for computing recursively defined functions

Ning, Q., van Dongen, V. and Gao, G.R., Automatic data and computation decomposition for distributed-memory machines

Norman, M.G., Pelagatti, S. and Thanisch, P., On the complexity of scheduling with communication delay and contention

Oksa, G., Time-minimal linear systolic arrays for the toeplitz system of linear equations

Palis, M.A., Liou, J.-C., Rajasekaran, S., Shende, S. and Wei, D.S.L., Online scheduling of dynamic trees

Pavel, S. and Akl, S.G., Efficient algorithms for the euclidean distance transform

Pelagatti, S., see Norman, M.G.

Pelc, A., Almost-safe Byzantine agreement in sparse networks with random faults

Perumalla, K. and Deo, N., Parallel algorithms for maximum subsequence and maximum subarray

Picquendar, M., Rosenberg, A.L. and Scarano, V., A cost-effective streamlining of the diogenes design methodology

Pölt, S., see Bollig, B.

Qiao, C., On designing communication-intensive algorithms for a spanning optical bus based array

Quinton, P., see Andonov, R.

Rajasekaran, S., see Palis, M.A.

Rajopadhye, S., see Andonov, R.

Ramaswamy, S. and Banerjee, P., Simultaneous allocation and scheduling using convex programming techniques

Rangan, C.P., see Mahesh, S.

Ranka, S., see Wang, J.-C.

Raynal, M., see Ahuja, M.

Richards, D., see Ibarra, L.

Risset, T., see Collard, J.-F.

Rosenberg, A.L., see Picquendar, M.

5 (1995) 15-22

5 (1995) 343-355

5 (1995) 437-449

5 (1995) 223-230

5 (1995) 387-399

5 (1995) 401-412

5 (1995) 1-2

5 (1995) 343-355

5 (1995) 111-124

5 (1995) 23-35

5 (1995) 149-156

5 (1995) 299-309

5 (1995) 539-550

5 (1995) 331-341

5 (1995) 461-474

5 (1995) 635-646

5 (1995) 205-212

5 (1995) 331-341

5 (1995) 489-497

5 (1995) 367-373

5 (1995) 513-524

5 (1995) $275-280$

5 (1995) 499-511

5 (1995) 251-262

5 (1995) 635-646

5 (1995) 251-262

5 (1995) 587-598

5 (1995) 343-355

5 (1995) 647-658

5 (1995) 171-178

5 (1995) 129-138

5 (1995) 421-436

5 (1995) 513-524 
Rosenberg, A.L., Vittorio Scarano and Sitaraman, R.K., The reconfigurable ring of processors: Efficient algorithms via hypercube simulation

Rossmanith, P., see Niedermeier, R.

Rover, D.T., see Chu, Y.-K.

Sadayappan, P., see Kumar, B.

Sahni, S., Data manipulation on the distributed memory bus computer

Saltz, J., see Chong, F.T.

Savický, P., see Bollig, B.

Scarano, V., see Picquendar, M.

Schenfeld, E., see Gupta, V.

Schröder, H., see Kaufmann, M.

Schuster, A., see Miller, $\mathrm{R}$

Seznec, A., see Drach, N.

Sharma, S.D., see Chong, F.T.

Shende, S., see Palis, M.A.

Shirazi, B., Youn, H.Y. and Lorts, D.M., Evaluation of static scheduling heuristic for real-time multiprocessing

Sibeyn, J.F., see Kaufmann, M.

Sinharoy, B., see Szymanski, B.K.

Sitaraman, R.K., see Rosenberg, A.L.

Skillicorn, D.B., see Cai, W.

Stalzer, M.A., A parallel fast multipole method for the Helmholtz equation

Suel, T., Permutation routing and sorting on meshes with row and column buses

Szymanski, B.K., Maniatty, W. and Sinharoy, B., Simultaneous parallel reduction on SIMD machines

Tel, G., Linear election in hypercubes

Thanisch, P., see Norman, M.G.

Tourancheau, B., see Desprez, F.

Tsai, W.T., see Bhattacharya, S.

van de Geijn, R., see Watts, J.

van Dongen, V., see Ning, Q.

Vittorio Scarano, see Rosenberg, A.L.

Vivien, F., see Darte, A.

Wang, J.-C., Lin, T.-H. and Ranka, S., Distributed scheduling of unstructured collective communication on the CM-5

Watts, J. and van de Geijn, R., A pipelined broadcast for multidimensional meshes

Wei, D.S.L.,

Wilde, D., see Andonov, R.

Wolski, R., Static scheduling of hierarchical program graphs

$\mathrm{Wu}, \mathrm{J}$., Tight bounds on the number of $I$-nodes in a faulty hypercube

Wu, M.-Y., Parallel incremental scheduling

Yang, T., see Gerasoulis, A.

Youn, H.Y., see Shirazi, B.

Ziavras, S.G., Scalable multifolded hypercubes for versatile parallel computers

5 (1995) $37-48$

5 (1995) 299-309

5 (1995) 623-634

5 (1995) 685-696

5 (1995) 3-14

5 (1995) 671-683

5 (1995) 275-280

5 (1995) 513-524

5 (1995) 563-574

5 (1995) 81-95

5 (1995) 1-2

5 (1995) 475-487

5 (1995) 671-683

5 (1995) 635-646

5 (1995) 599-610

5 (1995) 81-95

5 (1995) 437-449

5 (1995) 37-48

5 (1995) 179-190

5 (1995) 263-274

5 (1995) 63-80

5 (1995) 437-449

5 (1995) 357-366

5 (1995) 331-341

5 (1995) 157-169

5 (1995) 231-240

5 (1995) 281-292

5 (1995) 539-550

5 (1995) 37-48

5 (1995) 551-562

5 (1995) 647-658

5 (1995) 281-292

5 (1995) 635-646

5 (1995) 251-262

5 (1995) 611-622

5 (1995) 321-328

5 (1995) 659-670

5 (1995) 525-526

5 (1995) 599-610

5 (1995) 241-250 